JOURNAL OF

SYMPLECTIC GEOMETRY

Volume 1 Number 4, 683-693, 2002

\title{
AN EQUIVARIANT LIAPUNOV STABILITY TEST AND THE ENERGY-MOMENTUM-CASIMIR METHOD
}

\author{
ERIC T. MATsui
}

We present an equivariant Liapunov stability criterion for dynamical systems with symmetry. This result yields a simple proof of the energy-momentum-Casimir stability analysis of relative equilibria of equivariant Hamiltonian systems.

\section{Introduction}

We present an equivariant Liapunov stability criterion for dynamical systems with symmetry. This result yields a simple proof of the energymomentum-Casimir (EMC for short) stability analysis of relative equilibria of equivariant Hamiltonian systems.

The equivariant Liapunov stability test can be viewed as the natural adaptation of the classic Liapunov stability theorem to relative equilibria of equivariant dynamical systems. The proof echoes the traditional proof of Liapunov stability with all objects replaced by their equivariant/invariant counterparts.

The Liapunov stability theorem has numerous applications to Hamiltonian systems, where the Hamiltonian and other conserved quantities provide the ingredients of the Liapunov functions. 
The EMC method is widely used in applications to obtain sufficient conditions for group-stability of relative equilibria. The two key components of the EMC method are the construction of a suitable augmentation of the Hamiltonian and the application of a second derivative test (or, in the infinite-dimensional case, a convexity estimate) to establish stability.

The EMC method has been developed in a series of papers. The earliest version yielding rigorous group stability results used convexity arguments for systems on vector spaces; see [HMRW 85] and references therein. For free actions on manifolds, the result has been obtained by Patrick, [Pat92]. Non-free actions (with $G_{\mu}$ abelian) have been treated by Lewis, [Lew92]. The most general result to date (proper actions, $G_{\mu}$ compact) is due to Ortega, [Ort98].

In Section 3 we show that the application of the equivariant Liapunov stability test to this augmented Hamiltonian immediately yields Ortega's result. This shows that the only aspect of the Hamiltonian structure utilized in the stability analysis is the existence of an invariant conserved quantity. In particular, the analysis does not make use of the symplectic or Poisson structure.

In the last section, we present an extension of our results to infinitedimensional systems. We adapt the key ideas behind Arnol'd's method of convexity estimates ([Arn66, HMRW85]) to the group-equivariant setting.

These results comprise a part of the author's Ph.D. thesis ([Mat01a]). An application of the EMC method to the stability and bifurcation analysis of the sleeping pseudo-Lagrange top also appears in [Mat01a]. This application is further developed in [Mat01b] including an interesting example of a symmetry-breaking bifurcation to a family of steadily precessing motions where no "exchange of stability" occurs; both symmetric and asymmetric branches are stable in the neighborhood of the bifurcation point.

\section{Equivariant Liapunov Stability}

Let $X$ be a smooth vector field on a smooth finite-dimensional manifold $M$ that is equivariant with respect to a smooth and proper action of some Lie group $G$. Let $\mathfrak{g}$ denote the Lie algebra of $G$, and let $F_{t}, t \in \mathbb{R}$, denote the flow of $X$.

An element $m \in M$ is called a relative equilibrium if the curve $(\exp t \xi) \cdot m$ is a trajectory of $X$ for some $\xi$ in $\mathfrak{g}$. 
Let $G^{\prime}$ be a subgroup of $G$. A relative equilibrium $m$ of $X$ is said to be $G^{\prime}$-stable, or stable modulo $G^{\prime}$, if for every $G^{\prime}$-invariant open neighborhood $U$ of $G^{\prime} \cdot m$ there exists an open neighborhood $V$ of $m$ in $U$ such that $F_{t}(V) \subseteq U$ for all $t \geq 0$.

Theorem 1 (The Equivariant Liapunov Stability Test). Let $m \in M$ be a relative equilibrium. If there exists a $G$-invariant function $f$ such that

LS1. $m$ is a critical point of $f($ say, $f(m)=0)$,

LS2. the bilinear form $D^{2} f(m)$ is positive semi-definite with kernel $\mathfrak{g} \cdot m$, and

LS3. for every $\epsilon>0$ there exists an open neighborhood $V_{\epsilon}$ of $m$ such that

$$
f\left(F_{t}\left(V_{\epsilon}\right)\right) \subset[0, \epsilon), \quad \forall t>0,
$$

then $m$ is $G$-stable.

Proof. Without loss of generality, we may assume that $f(m)=0$.

Let $U$ be a $G$-invariant open neighborhood of the orbit $G \cdot m$. Our task is to construct an open neighborhood $V$ of $m$ such that $F_{t}(V) \subseteq U$ for all $t>0$.

By the Slice Theorem (see, e.g., $[\mathbf{G G K}]$ ), there exists a $G$-invariant open neighborhood $\tilde{M}$ of $G \cdot m$ and a $G$-equivariant projection map $\pi: \tilde{M} \rightarrow G \cdot m$ that makes $\tilde{M}$ a disc bundle. Let $W$ denote the fiber $\pi^{-1}(m)$.

Properties LS1, LS2, and the Morse Lemma guarantee the existence of positive number $\epsilon$ such that the connected component $W_{\epsilon}$ of $\left(\left.f\right|_{W}\right)^{-1}[0, \epsilon)$ is an open disk embedded in $W$ and contained in $U$. Let $G^{0}$ denote the connected component of $G$ containing the identity. Since $U$ is $G$-invariant, $G^{0} \cdot W_{\epsilon}$ is contained in $U$. Using the associated bundle structure of $\tilde{M}$ (see [GGK]), one can show that $G^{0} \cdot W_{\epsilon}$ is open and is the connected component of $f^{-1}[0, \epsilon)$ containing $m$.

Let $V=\left(G^{0} \cdot W_{\epsilon}\right) \cap V_{\epsilon}$, and let $v$ be an element of $V$. Since $m$ is an element of $V$, LS3 implies that $F_{t}(v)$ is in $[0, \epsilon)$ for all $t>0$, so that $F_{t}(v)$ is in the connected component of $f^{-1}[0, \epsilon)$ containing $v$, and, hence, $m$. This implies that $F_{t}(v)$ is in $G^{0} \cdot W_{\epsilon}$ for all $t>0$. Since $G^{0} \cdot W_{\epsilon} \subseteq U$, this proves that $m$ is $G$-stable.

\section{Application to Hamiltonian Systems with Symmetry}

Let $(M,\{\cdot, \cdot\})$ be a finite-dimensional Poisson manifold. Let $G$ act on $M$ properly and canonically. Let us assume that there exists an 
equivariant momentum map $\mathbf{J}: M \rightarrow \mathfrak{g}^{*}$ associated to the $G$-action. Let $H$ be a $G$-invariant Hamiltonian and $X_{H}$ its Hamiltonian vector field. Let $z_{e} \in M$ be a relative equilibrium of $X_{H}$ satisfying $X_{H}\left(z_{e}\right)=$ $\xi_{M}\left(z_{e}\right)$, where $\xi_{M}$ denotes the infinitesimal action on $M$ by some Lie algebra element $\xi \in \mathfrak{g}$. Set $\mu=\mathbf{J}\left(z_{e}\right)$.

Let us assume that there exist $G_{\mu}$-invariant norms $\|\cdot\|_{\mathfrak{g}}$ and $\|\cdot\|_{\mathfrak{g}^{*}}$ on $\mathfrak{g}$ and $\mathfrak{g}^{*}$, respectively, that satisfy

$$
\langle\alpha, \zeta\rangle \leq\|\alpha\|_{\mathfrak{g}^{*}}\|\zeta\|_{\mathfrak{g}}
$$

for all $\alpha \in \mathfrak{g}^{*}, \zeta \in \mathfrak{g}$. For example, this is the case if $G_{\mu}$ is compact. Let $V$ be an inner product space, and let $\mathbf{C}: M \rightarrow V$ be a map that is invariant with respect to the $G_{\mu}$-action and the flow. An energymomentum-Casimir function is defined by

$$
\mathcal{H}(z):=H(z)-J_{\xi}(z)+C_{\lambda}(z), \quad z \in M
$$

where $J_{\xi}(z):=\langle\mathbf{J}(z), \xi\rangle$ and $C_{\lambda}(z):=\langle\lambda, \mathbf{C}(z)\rangle$ for some $\lambda \in V^{*}$.

Theorem 2 (Energy-Momentum-Casimir Method, [Lew92, Ort98, Pat92]). Suppose that there exists a $\lambda$ in $V^{*}$ such that the following conditions hold:

EM1. $z_{e}$ is a critical point of $\mathcal{H}$.

EM2. The restriction of the bilinear form $D^{2} \mathcal{H}\left(z_{e}\right)$ to

$$
K=\operatorname{ker} D \mathbf{J}\left(z_{e}\right) \cap \operatorname{ker} D \mathbf{C}\left(z_{e}\right)
$$

is positive or negative semi-definite with kernel $\mathfrak{g}_{\mu} \cdot z_{e}$.

EM3. $G_{z_{e}} \subseteq G_{\xi}$.

Then $z_{e}$ is $G_{\mu}$-stable.

REMARK. The condition EM3 is trivially satisfied if the $G$-action is free, since $G_{z_{e}}$ would be trivial. If $G_{z_{e}}$ is nontrivial, then $\xi$ satisfying $X_{H}\left(z_{e}\right)=\xi_{M}\left(z_{e}\right)$ is determined up to $\mathfrak{g}_{z_{e}}$. Then $\xi$ can always be chosen so that EM3 is satisfied. The argument is as follows.

Since $G_{z_{e}}$ is compact, there exists a $G_{z_{e}}$-invariant complement $\mathfrak{g}_{z_{e}}^{\perp}$ to $\mathfrak{g}_{z_{e}}$ in $\mathfrak{g}$. Let $\xi^{\perp}$ be the $\mathfrak{g}_{z_{e}}^{\perp}$-component of $\xi$. By the equivariance of $X_{H}$, we have $g \cdot X_{H}\left(z_{e}\right)=X_{H}\left(g \cdot z_{e}\right)=X_{H}\left(z_{e}\right)$ for $g$ in $G_{z_{e}}$. Since $X_{H}\left(z_{e}\right)=$ $\xi_{M}\left(z_{e}\right)=\xi^{\perp}{ }_{M}\left(z_{e}\right)$, this implies that $g \cdot \xi^{\perp}{ }_{M}\left(z_{e}\right)=\left(\operatorname{Ad}_{g} \xi^{\perp}\right)_{M}\left(z_{e}\right)=$ $\xi^{\perp} M\left(z_{e}\right)$. Hence, $\operatorname{Ad}_{g} \xi^{\perp}-\xi^{\perp}$ is in $\mathfrak{g}_{z_{e}}$. On the other hand, $\mathfrak{g}_{z_{e}}^{\perp}$ is $G_{z_{e}}$-invariant, so that $\operatorname{Ad}_{g} \xi^{\perp}-\xi^{\perp}$ is in $\mathfrak{g}_{z_{e}}^{\perp}$. Therefore, $\operatorname{Ad}_{g} \xi^{\perp}=\xi^{\perp}$, and $G_{z_{e}} \subseteq G_{\xi^{\perp}}$. The algebra element $\xi$ satisfying $X_{H}\left(z_{e}\right)=\xi_{M}\left(z_{e}\right)$ and EM3 is not necessarily unique. This is important, because the difference in the choice of $\xi$ influences the validity of EM2. The relative 
equilibrium is stable as long as EM1-3 are satisfied for at least one choice of $\xi$, and having more choices increases the chance of establishing stability.

A case when EM3 is valid for all $\xi$ satisfying $X_{H}\left(z_{e}\right)=\xi_{M}\left(z_{e}\right)$ is when $G_{\mu}$ is abelian, which is generically the case if $G_{\mu}$ is connected (see [DV69, MR94]). In this case, since $\xi$ is an element of $G_{\mu}$, it follows that $G_{\mu} \subseteq G_{\xi}$. Since $G_{z_{e}}$ is contained in $G_{\mu}$, EM3 is satisfied.

Proof. We first prove the case when $D^{2} \mathcal{H}\left(z_{e}\right)$ is positive semi-definite on $K$.

The condition EM3 is necessary for the following construction. By the Slice Theorem, there exists a $G_{\mu}$-invariant open neighborhood $\tilde{M}$ of $z_{e}$ and a $G_{\mu}$-equivariant projection map $\pi: \tilde{M} \rightarrow G_{\mu} \cdot z_{e}$ that makes $\tilde{M}$ a vector bundle. Let $\phi: G_{\mu} / G_{z_{e}} \rightarrow G_{\mu} \cdot z_{e}$ denote the natural isomorphism. Define $\tilde{\pi}: \tilde{M} \rightarrow G_{\mu} / G_{z_{e}}$ as $\tilde{\pi}=\phi^{-1} \circ \pi$. The condition EM3 guarantees that

$$
\operatorname{Ad}_{\tilde{\pi}(z)} \xi, \quad z \in M
$$

is well defined. This is called Patrick's velocity map ([Ort98]). We incorporate it in the definition of the $G_{\mu}$-invariant function used in the proof of the equivariant Liapunov stability test. Let

$$
f(z)=f_{1}(z)+\sigma f_{2}(z), \quad z \in M,
$$

where $\sigma$ is a real number sufficiently large so that $f$ satisfies LS2 (see below), and $f_{1}$ and $f_{2}$ are defined by

$$
\begin{aligned}
& f_{1}(z)=H(z)-\left\langle\mathbf{J}(z), \cdot \operatorname{Ad}_{\tilde{\pi}(z)} \xi\right\rangle+C_{\lambda}(z) \\
& f_{2}(z)=\|\mathbf{J}(z)-\mu\|_{\mathfrak{g}^{*}}^{2}+\left\|\mathbf{C}(z)-\mathbf{C}\left(z_{e}\right)\right\|_{V}^{2} .
\end{aligned}
$$

The $G_{\mu}$-equivariance of $\mathbf{J}$ and $\tilde{\pi}$ guarantees that $f_{1}$ is $G_{\mu}$-invariant. The $G_{\mu}$-invariance of $f_{2}$ follows from the equivariance of $\mathbf{J}$ and $G_{\mu^{-}}$ invariance of $\|\cdot\|_{\mathfrak{g}^{*}}$ and $\mathbf{C}$. Hence, $f$ is $G_{\mu}$-invariant.

We now prove that $f$ satisfies conditions LS1, LS2, and LS3, for sufficiently large values of $\sigma$.

Condition $L S 1$. That $z_{e}$ is a critical point of $f_{2}$ is obvious. Hence, it suffices to prove that $z_{e}$ is a critical point of $f_{1}$.

Let $S$ denote the slice $\pi^{-1}\left(z_{e}\right)$. Then

$$
T_{z_{e}} M=\left(\mathfrak{g}_{\mu} \cdot z_{e}\right) \oplus\left(T_{z_{e}} S\right) .
$$

Since $f_{1}$ is $G_{\mu}$-invariant, this implies that we only need to show that $z_{e}$ is a critical point of $\left.f_{1}\right|_{S}$. Since $\tilde{\pi}(S)=\phi^{-1}\left(z_{e}\right)=G_{z_{e}} \subseteq G_{\xi}$ (where 
$\phi: G_{\mu} / G_{z_{e}} \rightarrow G_{\mu} \cdot z_{e}$ is the natural isomorphism) we have $\operatorname{Ad}_{\tilde{\pi}(z)} \xi=\xi$ for $z$ in $S$, so that

$$
f_{1}(z)=\mathcal{H}(z), \quad \text { for } z \in S .
$$

Therefore, by EM1, $z_{e}$ is a critical point of $\left.f_{1}\right|_{S}$. This proves that $z_{e}$ is a critical point of $f=f_{1}+\sigma f_{2}$.

Condition LS2. From the decomposition (3), we see that the condition LS2 is satisfied if and only if $D^{2} f\left(z_{e}\right)$ is positive-definite on $T_{z_{e}} S$. Let $D_{S}^{2} f_{1}\left(z_{e}\right)$ and $D_{S}^{2} f_{2}\left(z_{e}\right)$ denote the restrictions of $D^{2} f_{1}\left(z_{e}\right)$ and $D^{2} f_{2}\left(z_{e}\right)$ to $T_{z_{e}} S$, respectively. We claim that $D_{S}^{2} f_{2}\left(z_{e}\right)$ is positive semi-definite with kernel $K \cap T_{z_{e}} S$, and that $D_{S}^{2} f_{1}\left(z_{e}\right)$ is positivedefinite on $K \cap T_{z_{e}} S$. Then $D_{S}^{2} f_{1}\left(z_{e}\right)+\sigma D_{S}^{2} f_{2}\left(z_{e}\right)$ is positive-definite for sufficiently large values of $\sigma$, so that $f$ satisfies LS2.

The claim is proved as follows.

Since $D^{2} f_{2}\left(z_{e}\right)$ is positive semi-definite with kernel $K$, we conclude that the quadratic form $D_{S}^{2} f_{2}\left(z_{e}\right)$ is positive semi-definite with kernel $K \cap T_{z_{e}} S$.

Since $K$ contains $\mathfrak{g}_{\mu} \cdot z_{e}$, the decomposition (3) implies that

$$
K=\left(\mathfrak{g}_{\mu} \cdot z_{e}\right) \oplus\left(K \cap T_{z_{e}} S\right) .
$$

Therefore, by EM2, $D^{2} \mathcal{H}\left(z_{e}\right)$ is positive-definite on $K \cap T_{z_{e}} S$. Combining this with $(4)$, we conclude that $D_{S}^{2} f_{1}\left(z_{e}\right)$ is positive-definite on $K \cap T_{z_{e}} S$.

Condition LS3. Let $F_{t}$ denote the flow of $X_{H}$. Since $\tilde{\pi}\left(z_{e}\right)=G_{z_{e}} \subseteq$ $G_{\xi}$, we have $\left\langle\mathbf{J}\left(z_{e}\right), \operatorname{Ad}_{\tilde{\pi}\left(z_{e}\right)} \xi\right\rangle=\mu \cdot \xi$, and

$$
\begin{aligned}
f\left(F_{t}(z)\right)-f\left(z_{e}\right)= & H\left(F_{t}(z)\right)-H\left(z_{e}\right)-\left(\left\langle\mathbf{J}\left(F_{t}(z)\right), \operatorname{Ad}_{\tilde{\pi}\left(F_{t}(z)\right)} \xi\right\rangle-\langle\mu, \xi\rangle\right) \\
& +C_{\lambda}\left(F_{t}(z)\right)-C_{\lambda}\left(z_{e}\right)+\sigma f_{2}\left(F_{t}(z)\right) \\
= & H(z)-H\left(z_{e}\right)-\left(\left\langle\mathbf{J}(z), \operatorname{Ad}_{\tilde{\pi}\left(F_{t}(z)\right)} \xi\right\rangle-\langle\mu, \xi\rangle\right) \\
& +C_{\lambda}(z)-C_{\lambda}\left(z_{e}\right)+\sigma f_{2}(z) .
\end{aligned}
$$

Since $\tilde{\pi}(z)$ is in $G_{\mu} / G_{z_{e}}$ for any $z \in \tilde{M}$, we have $\mu=\operatorname{Ad}_{\tilde{\pi}\left(F_{t}(z)\right)}^{*} \mu$, and

$$
\begin{aligned}
f\left(F_{t}(z)\right)-f\left(z_{e}\right)= & H(z)-H\left(z_{e}\right)-\left\langle\mathbf{J}(z)-\mu, \operatorname{Ad}_{\tilde{\pi}\left(F_{t}(z)\right)} \xi\right\rangle \\
& +C_{\lambda}(z)-C_{\lambda}\left(z_{e}\right)+\sigma f_{2}(z) \\
\leq & \left|H(z)-H\left(z_{e}\right)\right|+\left\|\mathbf{J}(z)-\mathbf{J}\left(z_{e}\right)\right\|_{\mathfrak{g}^{*}}\|\xi\|_{\mathfrak{g}} \\
& +\left|C_{\lambda}(z)-C_{\lambda}\left(z_{e}\right)\right|+\sigma\left|f_{2}(z)\right| .
\end{aligned}
$$

The inequality follows from (1) and $G_{\mu}$-invariance of $\|\cdot\|_{\mathfrak{g}}$. Hence, LS3 is satisfied by the continuity of $H, \mathbf{J}$, and $\mathbf{C}$. 
If $D^{2} \mathcal{H}\left(z_{e}\right)$ is negative semi-definite on $K$ with kernel $\mathfrak{g}_{\mu} \cdot z_{e}$, then we redefine $f$ by

$$
f(z)=-f_{1}(z)+\sigma f_{2}(z), \quad z \in M .
$$

The proof proceeds as in the positive semi-definite case.

\section{Extension to Infinite Dimensions}

It is well-known that the positive-definiteness of the Hessian of the energy at an equilibrium is a sufficient condition for its stability, provided that the system is finite-dimensional. For infinite-dimensional systems, additional assumptions are necessary to establish stability. The convexity estimate method due to Arnol'd has been widely used to circumvent this shortcoming in order to establish nonlinear stability. (See, e.g., [HMRW 85], and references therein.) In the remainder of this article, we present a possible adaptation of Arnol'd's approach to the stability of relative equilibria with isotropy in infinite dimensions. We make use of the Slice Theorem for the action of compact groups on Banach manifolds, which allows us to easily extend the results of the previous two sections to this setting. (See, e.g., $[\mathbf{G G K}]$ for the precise statement of the Slice Theorem on Banach manifolds.)

4.1. Equivariant Liapunov Stability. Let $M$ be a Banach manifold and let $G$ be a compact Lie group acting on $M$. Let $X$ be a $G$-equivariant vector field on $M$. Note that we do not assume that $X$ is Lipschitz or even continuous. Relative equilibria of $X$ can still be defined in the usual way. (However, without suitable smoothness assumptions on $X$ we cannot guarantee existence and uniqueness of an integral curve for any initial conditions.)

Theorem 3. Let $m$ be a relative equilibrium. Fix the Slice Theorem decomposition $G \times{ }_{G_{m}} S$ of a neighborhood of $G \cdot m$, where $S$ is an open ball in a Banach space. Without loss of generality, we may assume that the norm on $S$ is $G_{m}$-invariant. Let $P: M \rightarrow \mathbb{R}$ be a continuous $G$-invariant function whose restriction to $S$ is a function of the norm which has a strict minimum (say, equal to zero) at the origin. If there exists a $G$-invariant function $f$, with $f(m)=0$, such that

LS1'. $m$ is a critical point of $f$,

$\mathrm{LS} 2^{\prime} . P(u) \leq f(u)$ for all $u$ near $m$, and

LS3'. for every $\epsilon>0$ there exists a neighborhood $V$ of $m$ such that $f(u(t))<\epsilon$ for any integral curve $u(t)$ with initial condition $u(0)$ in $V$. 
Then for all $\epsilon>0$ there exists a neighborhood $U$ of the orbit such that $P(u(t))<\epsilon$ for any integral curve $u(t)$ with $u(0)$ in $U$ as long as $u(t)$ is defined.

The proof of this theorem is essentially identical to the proof of the Theorem 1. The role of condition LS2 $2^{\prime}$ is to ensure that $f^{-1}[0, \epsilon)$ contains a small neighborhood of the orbit $G \cdot m$. (In the finite-dimensional version, this follows from LS2 and the Morse Lemma.)

REMARK.

1) By the Slice Theorem, $P$ only needs to be defined on the slice through $m$.

2) If $P$ is the square of the original Banach norm on the slice, we recover the $G$-stability as in $\S 2$. Sometimes, $P$ is not even the square of a norm; see [Lew94, Hes80].

3) With suitable modifications, the theorem can be extended to the case where $P$ is a function of a norm which is weaker than the original norm on $S$. This generalization is useful when the original norm is chosen to be as close as possible to guaranteeing the existence and uniqueness of solutions and the "norm square" $P$ is defined by the Hessian of $f$ (cf. [Bru02, HMRW85]).

4.2. The EMC method. Let $M$ be a smooth Banach Poisson manifold and let $G$ be a compact Lie group that acts on it canonically. In the following theorem, we let $S$ be the $G_{\mu}$-slice through $z_{e}$. By the Slice Theorem, we can think of $S$ as a ball, centered at the origin, in a Banach space. Let $\mathcal{H}$ and $f_{2}$ be defined as before. Set $\mathcal{H}_{\sigma}=\mathcal{H}+\sigma f_{2}$.

Theorem 4. Suppose that $H, \mathbf{J}$, and $\mathbf{C}$ are continuous and there exists $\lambda \in V^{*}$ and $\sigma \in \mathbb{R}$ such that the following conditions hold:

EM1'. $z_{e} \in M$ is a critical point of $\mathcal{H}$.

EM2'. There exists a real number $C$ such that

$$
\|\Delta z\|^{2} \leq D^{2} \mathcal{H}_{\sigma}(z)(\Delta z, \Delta z)
$$

for all $z$ in $S$ near the origin and for all $\Delta z$ in $S$.

EM3'. $G_{z_{e}} \subseteq G_{\xi}$.

Then $z_{e}$ is Liapunov stable in the sense of Theorem 3.

This theorem is proved by using Theorem 3 in the same way as Theorem 1. Note that $\mathcal{H}_{\sigma}$ and $f=f_{1}+\sigma f_{2}$ (as defined in the proof of the finite-dimensional EMC theorem) are identical on $S$. The difference is that $f$ is globally $G_{\mu}$-invariant, while $\mathcal{H}_{\sigma}$ is not. Hence, we apply 
Theorem 3 to $f$, not $\mathcal{H}_{\sigma}$. The condition LS2' follows from EM2' by the Mean Value Theorem.

ACKnowledgements. The author would like to thank Viktor Ginzburg and Debra Lewis for their invaluable support during the preparation of this article. 


\section{Bibliography}

[Arn66] V.I. Arnol'd, An a priori estimate in the theory of hydrodynamic stability, Izv. Vysš. Učebn. Zaved. Matematika 1966 (1966), no. 5 (54), 3-5.

[Bru02] J.K. Bruschi, Bifurcations of whirling states of an elastic string, Ph.D. thesis, University of California, Santa Cruz, 2002 , in preparation.

[DV69] M. Duflo and M. Vergne, Une proprieté de la représentation coadjointe d'une algébre de Lie, C.R. Acad. Sci. Paris 268 (1969), 583-585.

[GGK] V.L. Ginzburg, V. Guillemin, and Y. Karshon, Moment maps, cobordisms, and hamiltonian group actions, in preparation.

[Hes80] M.R. Hestenes, Calculus of variations and optimal control theory, Robert E. Krieger Publishing Co. Inc., Huntington, N.Y., 1980, Corrected reprint of the 1966 original.

[HMRW85] D.D. Holm, J.E. Marsden, T.S. Ratiu, and A. Weinstein, Nonlinear stability of fluid and plasma equilibria, Phys. Rep. 123 (1985), 1-116.

[Lew92] D. Lewis, Lagrangian block diagonalization, J. Dynamics Differential Equations 4 (1992), 1-41.

[Lew94] D. Lewis, Quasi-periodic motions of planar liquid drops, Differential geometry and mathematical physics (Vancouver, BC, 1993), Amer. Math. Soc., Providence, RI, 1994, pp. 185-199.

[Mat01a] E.T. Matsui, Bifurcation and stability of relative equilibria with isotropy in lagrangian systems with symmetry, Ph.D. thesis, University of California, Santa Cruz, 2001.

[Mat01b] E.T. Matsui, Stability-preserving bifurcations of the relative equilibria, In preparation, 2001.

[MR94] J.E. Marsden and T.S. Ratiu, Introduction to mechanics 
and symmetry, Texts in Applied Mathematics, vol. 17, Springer-Verlag, 1994.

[Ort98] J.P. Ortega, Symmetry, reduction, and stability in hamiltonian systems, Ph.D. thesis, University of California, Santa Cruz, 1998.

[Pat92] G.W. Patrick, Relative equilibria in Hamiltonian systems: the dynamic interpretation of nonlinear stability on a reduced phase space, J. Geom. Phys 9 (1992), 111-119.

Department of Mathematics and Statistics, University of Ottawa, 585

King Edward Ave., Ottawa, ON K1N 6N5, Canada

E-mail address: ematsui@uottawa.ca 